\title{
Fundamental Bounds on Downlink Throughput of Cellular Networks
}

\author{
Do Trong Hieu, Sang-Won Choi, Young-Seob Choi and Sae-Young Chung \\ Department of Electrical Engineering and Computer Science \\ Korea Advanced Institute of Science and Technology \\ E-mail: \{hieudt,ace1905,seovie\}@kaist.ac.kr, sychung@ee.kaist.ac.kr
}

\begin{abstract}
Throughput (or maximum sum rate) of the downlink in cellular networks has always been an issue of importance since it reveals the maximum capability of the networks. In this paper we employ an information-theoretic approach to establish upperbounds and lowerbounds on the throughput of the downlink in cellular networks. These bounds may serve as baselines for evaluating performance of practical transmission schemes. In a Multiple Input Multiple Output (MIMO) cellular architecture we use the zero forcing criterion to design the optimal number of antennas at each receiver given the number of antennas at each transmitter, thereby deriving a lowerbound for the total achievable multiplexing gain. This lowerbound is shown to meet the upperbound in certain cases.

Index Terms-Downlink throughput, multiplexing gain
\end{abstract}

\section{INTRODUCTION}

We investigate the downlink of a multi-cell network where there are multiple base stations and multiple mobile stations. Practically, this network model can be applied to the mobile cellular networks as well as wireless local area networks (wireless LANs). In the information theory literature this multiple-transmitter multiple-receiver scenario is termed the interference channel (IC). The capacity region of the interference channel is a notoriously difficult problem. Even the capacity region of the simple 2-input 2-output scalar interference channel is only known in limited cases [1]. Therefore, finding the outerbounds and innerbounds for the capacity region and for the sumrate of the interference channel has been drawing great interests from the community [2], [3]. In the early parts of this paper, we focus on characterizing the bounds on sumrate of the interference channel, thereby setting up upperbounds and lowerbounds on the downlink throughput of the multi-cell networks. The results provide a comprehensive view on the limits of throughput in such networks and may help to evaluate the performance of practical transmission schemes.

It is well known that capacity of an $M$ by $N$ MIMO point-to-point system increases linearly as $\min (M, N)$ at high signal to noise ratio (SNR). That scaling factor is basically the spatial degrees of freedom coming from having multiple antennas and is termed multiplexing gain [4]. For the MIMO multi-point, the multiplexing gain is interpreted as the scaling factor of the sumrate over $\log (S N R)$ at high SNR. Recently, Jafar et al. have characterized upperbound and lowerbound on the multiplexing gain of MIMO interference channel in 2-input 2-output case [5]. In a more recent work [6], Jafar considered the multiplexing gain of the MIMO X channel, which is physically a MIMO interference channel where each transmitter wishes to send different messages to multiple receivers. The author established lower and upperbound on the multiplexing gain of a MIMO X channel with 2 transmitters and 2 receivers. The result was the generalization of the scheme proposed by Maddah-Ali $e t$ al. [7] (the MMK scheme). In a later part of this paper, we explore the multiplexing gain of the downlink where the each base station sends different messages to mobile terminals in different cells. This is a MIMO X channel with a general number of transmitters and receivers. We use the zero forcing technique to find the optimal number of antennas at each receiver given the number of antenna at each transmitter and setup a lowerbound on the achievable multiplexing gain. We show that for the 2-transmitter 2-receiver channel with certain number of transmit antennas our result coincides with the maximum achievable multiplexing gain proved in [6].

The remaining of this paper is as follows: Section II formulates the problem. Section III setups the Multiple Access Channel upperbound (MAC-UB), the Broadcast Channel upperbound (BC-UB), and the Interference-Single User Detection lowerbound (IFSUD-LB), respectively. Section IV provides numerical results and analysis. Note that for the ease of analysis, we employ the single-antenna model in Sections III and IV. In Section V we broaden the problem to a Multiple Input - Multiple Output (MIMO) scenario where the mobile terminals can receive messages from different base stations and propose an achievable scheme (lowerbound) for the multiplexing gain of the network. The paper is concluded in Section VI.

\section{Problem formulation}

Consider a multi-cell network in which each cell has a perfect hexagonal shape and the base station is located at the center of the cell. For the sake of simplicity, we assume here a simplified network model (Fig. 1). In this model, there is a center cell surrounded by 6 neighboring cells. Each cell has one base station (BS) and one mobile station (MS) and the transmission in a cell is interfered by transmissions from all other cells. We assume both BS and MS are singleantenna stations and the antennas are omni directional. Each 
transmit antenna at the base stations is imposed with a power constraint $P_{i}$. Here we are considering the single-user-percell scenario, in a multi-user-per-cell scenario there will be the benefit of multiuser diversity gain.

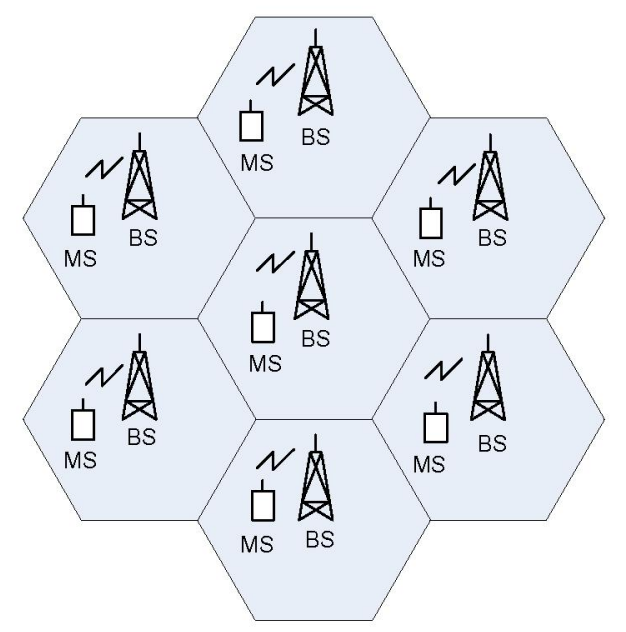

Fig. 1. Simplified network mode1

In the network, every MS receives its intended signal from the host BS and suffers from interference caused by neighboring BSs. We assume a block fading channel model, i.e., channels are constant in each time block but possibly change from one block to another. Noise at every receiver are assumed white Gaussian with zero mean and unit variance. Noise at different receivers are uncorrelated. We further assume the channel state information (CSI) is perfectly known at mobile stations as well as at base stations (full CSIT and CSIR). To this end the received signal at the MS in cell $k$ is given by

$$
y_{k}=h_{k k} x_{k}+\sum_{i \neq k} h_{i k} x_{i}+n_{k}
$$

where $x_{k}$ is the intended signal for MS $k, h_{i k}$ is the channel gain between BS $i$ and MS $k$. The term $\sum_{i \neq k} h_{i k} x_{i}$ denotes interference from adjacent cells to MS $k . n_{k}$ is white Gaussian noise at MS $k$. Throughput (or sum rate capacity) of the cellular network is defined as the maximum achievable sum rate of all MSs under the given power constraints.

$$
C_{N W}^{s u m} \triangleq \max _{\left\{Q_{i} \leq P_{i} \vee i\right\}} \sum_{i} R_{i}
$$

where $Q_{i}$ and $P_{i}$ are the transmit power and the power constraint at BS $i$, respectively. $R_{i}$ is the achievable rate at MS $i$.

In the next section we establish various bounds for the downlink throughput by considering different levels of cooperation in the network. For terminology, we will use MS, mobile station, mobile terminal interchangeably and similarly with BS, bases, and base stations.

\section{UPPERBOUNDS AND LOWERBOUND}

\section{A. MAC Upperbound (MAC-UB)}

Multiple Access Channel is a channel in which different users independently send their messages to a common receiver. The uplink in a wireless network can be modeled as a MAC channel. The capacity region of the MAC channel is well established (see [8] and references therein).

In the network under consideration, if we allow for the cooperation among mobile stations but not among the bases, the downlink of the network can be viewed as a Multiple Access Channel in which each BS acts as a user and all MSs together act as a single receiver. The cooperation obviously allows better processing of the received signal. Accordingly the capacity region and the sum rate capacity of this MAC can be taken as upperbounds for the capacity region and the throughput of the downlink in the network.

Consider a MIMO MAC channel with $K$ transmitters and one receiver. Let $\mathbf{x}_{k} \in \mathbb{C}^{r_{k} \times 1}$ denote the transmitted signal of transmitter $k$. Let $\mathbf{y} \in \mathbb{C}^{M \times 1}$ be the received signal and $\mathbf{n} \in$ $\mathbb{C}^{M \times 1}$ the noise vector where $\mathbf{n} \sim N(0,1)$. The received signal is represented as

$$
\begin{aligned}
\mathbf{y} & =\sum_{k=1}^{K} \mathbf{H}_{k} \mathbf{x}_{k}+\mathbf{n} \\
& =\mathbf{H x}+\mathbf{n}
\end{aligned}
$$

where $\mathbf{H}=\left[\mathbf{H}_{1} \ldots \mathbf{H}_{K}\right]$ and $\mathbf{x}=\left[\mathbf{x}_{1} \ldots \mathbf{x}_{K}\right]^{T}$

Each transmitter is imposed with an individual power constraint of $P_{i}$. The channel matrices $\mathbf{H}_{1}, \ldots, \mathbf{H}_{K}$ are assumed fixed and known at all the transmitter and receivers. The capacity region of this MIMO MAC is given by [9]:

$$
\begin{aligned}
& \mathcal{C}_{M A C}\left(\mathbf{H}_{1}, \ldots, \mathbf{H}_{K}, P_{1}, \ldots, P_{K}\right) \\
& \triangleq \bigcup_{\left\{\mathbf{Q}_{\mathbf{i}} \geq 0, \operatorname{Tr}\left(\mathbf{Q}_{i}\right) \leq P_{i} \quad \forall i\right\}}\left\{\left(R_{1}, \ldots, R_{K}\right):\right. \\
& \left.\sum_{i \in S} R_{i} \leq \log \left|\mathbf{I}+\sum_{i \in S} \mathbf{H}_{i} \mathbf{Q}_{i} \mathbf{H}_{i}^{\dagger}\right| \forall S \subseteq\{1, \ldots, K\}\right\}
\end{aligned}
$$

and the sum rate capacity of the MIMO MAC is defined as:

$$
\begin{aligned}
C_{M A C}^{\text {sum }} & \triangleq \max _{R_{1}, \ldots, R_{K} \in \mathcal{C}_{M A C}\left(\mathbf{H}_{1}, \ldots, \mathbf{H}_{K}, P_{1}, \ldots, P_{K}\right)} \sum_{i=1}^{K} R_{i} \\
& =\max _{\operatorname{Tr}\left(\mathbf{Q}_{i}\right) \leq P_{i}} \log \left|\mathbf{I}+\sum_{i=1}^{K} \mathbf{H}_{i} \mathbf{Q}_{i} \mathbf{H}_{i}^{\dagger}\right|
\end{aligned}
$$

where $\mathbf{Q}_{i}$ 's is the transmit covariance matrix of user $i$. Hence we have the following bound:

$$
C_{N W}^{s u m} \leq C_{M A C}^{s u m}
$$

where $C_{M A C}^{s u m}$ is sum rate capacity of the MAC formed by allowing the MSs to cooperate. We name this throughput upperbound MAC-UB. 


\section{B. BC upperbound (BC-UB)}

It is noted that recently there have been several works in the literature evaluating the performance of cooperation among base stations [10],[11]. In this paper, we are interested in establishing the fundamental bound of throughput.

Broadcast channel is a channel in which there is one transmitter sending private messages to different receivers (and possibly common messages to all users). The downlink of a cellular network can be modeled a BC channel. Consider a Multiple Input Multiple Output Gaussian Broadcast channel (MIMO-GBC) with one base station equipped with $M$ transmit antennas and $K$ users with $n_{r}$ receive antennas each. The received signal at user $k$ is

$$
\mathbf{y}_{k}=\mathbf{H}_{k} \mathbf{x}+\mathbf{n}_{k}
$$

where $\mathbf{x} \in \mathbb{C}^{M}, \mathbf{y}_{k} \in \mathbb{C}^{n_{r}}, \mathbf{H}_{k} \in \mathbb{C}^{n_{r} \times M}$ denote the transmitted signal at BS, the received signal, and the channel matrix of user $k$, respectively. $\mathbf{n}_{k} \sim \mathcal{C N}(0, \mathbf{I})$ represents the circularly symmetric Gaussian noise at user $k$. Recently it is proved that the sumrate capacity of MIMO-GBC can be achieved by a precoding scheme known as Dirty Paper Coding (DPC) [12],[13],[14],[15] and is given by

$$
\begin{aligned}
\mathcal{C}_{B C}^{\text {sum }} & =\mathcal{C}_{D P C}^{\text {sumrate }}\left(\mathbf{H}_{1}, \ldots, \mathbf{H}_{K}, P\right) \\
& =\max _{\sum_{i=1}^{K} \operatorname{Tr}\left(\boldsymbol{\Sigma}_{i}\right) \leq P, \boldsymbol{\Sigma}_{i} \geq 0} \log \frac{\left|\mathbf{I}+\mathbf{H}_{i}\left(\sum_{j \geq i} \boldsymbol{\Sigma}_{j}\right) \mathbf{H}_{i}^{\dagger}\right|}{\left|\mathbf{I}+\mathbf{H}_{i}\left(\sum_{j>i} \boldsymbol{\Sigma}_{j}\right) \mathbf{H}_{i}^{\dagger}\right|} \\
& =\max _{\mathbf{Q}_{i} \geq 0, \sum_{i=1}^{K} \operatorname{Tr}\left(\mathbf{Q}_{i}\right) \leq P} \log \left|\mathbf{I}+\sum_{i=1}^{K} \mathbf{H}_{i}^{\dagger} \mathbf{Q}_{i} \mathbf{H}_{i}\right|
\end{aligned}
$$

where (7) comes from the BC-MAC duality [13]. $\boldsymbol{\Sigma}_{i}$ and $\mathbf{Q}_{i}$ are the transmit covariance matrix for user $i$ in $\mathrm{BC}$ and the dual MAC, respectively. $P$ is power constraint at the $\mathrm{BC}$ transmitter. It is recently proved that the capacity region of the MIMO-GBC is also achieved by DPC with optimal beamforming and power allocation [16]. There are a number of algorithms to maximizing the sum rate and to find the capacity region of MIMO Gaussian BC [17][18][19].

In the simplified network model under consideration, if we allow for the cooperation among the base stations but not among the mobile stations the cellular network can be viewed as a vector broadcast channel. To set up the upperbound for the downlink throughput, we suppose the base stations are interconnected by a high speed backbone network so that the base stations can exchange their data instantly. Each mobile station can be served by all the base stations. The bases together act as a transmitter with $N_{b}$ transmit antennas where $N_{b}$ is the number of cooperating bases. Hence we have the following upperbound

$$
C_{N W}^{s u m} \leq C_{B C}^{\text {sum }}
$$

where $C_{B C}^{\text {sum }}$ is the sum rate capacity of the $\mathrm{BC}$ formed by allowing the bases to cooperate. We name this upperbound BC-UB. This bound is achieved by using DPC with optimal beamforming and power allocation at the cooperating base stations.

\section{Interference channel lowerbound (IFSUD-LB)}

Interference channel is an information-theoretic channel model in which there are multiple transmitters and multiple receivers. There is no cooperation and the transmissions from transmitters cause interference to each other.

In general, the capacity region of the interference channel is not known yet. However, we can develop a simple lowerbound for the capacity region as well as sum rate of an interference channel by considering the single user detection (SUD) at the receivers. In this scenario, each receiver detects his own signal by treating others' signals as interference. In general, a multi-cell wireless network can be modeled as an interference channel where each mobile station suffers from inter-cell interference. Thus, we can use the lowerbound for the sum rate of an interference channel as an lowerbound for throughput of the downlink in a cellular network.

In our simplified network model, the rate of user $i$ when performing SUD is given by

$$
R_{i}=\log \left(1+\frac{h_{i i}^{2} P_{i}}{1+\sum_{j \neq i} h_{i j}^{2} P_{j}}\right)
$$

and the sum rate is

$$
R_{I F S U D}^{\text {sum }}=\sum_{i=1}^{K} R_{i}
$$

where $h_{i j}$ is channel gain between MS $i$ and $\mathrm{BS} j$ and $P_{j}$ is transmit power at BS $j$. Then we have the following bound

$$
C_{N W}^{s u m} \geq R_{I F S U D}^{\text {sum }}
$$

We named this bound IFSUD-LB. In order to enhance system capacity, one can employ various multiuser detection (MUD) techniques such as minimum-mean-square-error (MMSE), successive interference cancelation (SIC) or even more sophisticated MUD techniques, see [20] and references therein.

\section{NUMERICAL RESUlTS AND ANALYSiS}

We present here the plots of above bounds in terms of average transmit power at each base station. The plots show MAC-UB, BC-UB and IFSUD-LB. We also plot the achievable sum rate if we allow the BSs to cooperate and perform Channel Inversion (Chan-Inv) and the achievable sum rate for conventional transmitting scheme like FDM or TDM with BS cooperation (FDM-TDM-coop) and FDM or TDM without BS cooperation (FDM-TDM-nocoop).

For simplicity, channel gain from a BS to an MS in the same cell is assumed to be 1, channel gains from a BS to MSs in adjacent cells are assumed to be equal and equal $\alpha$ with $0 \leq \alpha \leq 1$. The received signal at MS $k$ in (1) becomes

$$
\begin{aligned}
y_{k} \quad=x_{k}+\sum_{i \neq k} h_{i k} x_{i}+n_{k} \\
=x_{k}+\sum_{i \neq k} \alpha x_{i}+n_{k}
\end{aligned}
$$

For MAC-UB we impose the same power constraint for every BS, which is $P_{i} \leq 1$ for $i=1, \ldots, 7$. For BC-UB, ChanInv and FDM-TDM-coop we use the total power constraint 
equals to the sum power constraint in MAC-UB. Finally, the FDM-TDM-nocoop and IFSUD-LB is plotted with the same power constraint per each BS as in MAC-UB. The plot with $\alpha=0.5$ is shown in Fig. 2 .

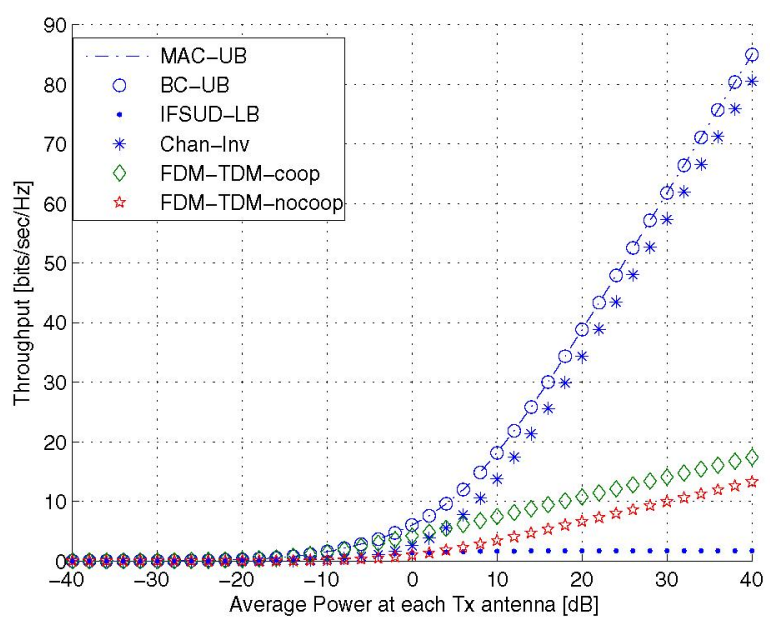

Fig. 2. Fundamental Bounds on Throughput of Cellular Network

As shown in the plot, the MAC-UB and BC-UB are identical. This can be explained by the MAC-BC duality [13], which states that the capacity region of a MIMO broadcast channel with power constraint $P$ is equal to the capacity region of the dual MAC with sum power constraint $P$, and the fact that the channel is scalar in this case. The plot also reveals the multiplexing gain of 7 of the BC-UB, which equals the number of transmit antennas at the transmitter when the BSs can cooperate. The channel inversion scheme, although has a certain constant performance gap compared with the BC-UB high SNR, has the same multiplexing gain of 7 (the slope of the curve at high SNR) because channel inversion essentially diagonalizes the channel matrix. For the IFSUD-LB, the curve soon becomes saturated as the transmit power at the bases increase since the channel of each MS is interference-limited. The multiplexing gain is equal to 0 . The plot of FDM-TDM-nocoop and FDM-TDM-coop both show the multiplexing gain of 1 , which come from the orthogonal nature of those schemes. However, there is a gap between FDM-TDM-nocoop and FDM-TDM-coop, which is the power gain. It is also noticed that at low SNR (power limited regime), FDM or TDM is optimal.

In summary, by allowing cooperation among the BSs or among the MSs, the downlink throughput increases linearly with number of antennas at high SNR. Not allowing any cooperation and just perform SUD at the MSs results in bad performance and should be avoided at high SNR. Conventional schemes like TDM or FDM always give a multiplexing gain of 1 and is optimal at low SNR. Allowing partial cooperation or performing suboptimal precoding or beamforming may achieve somewhere between the above upperbounds and the lowerbounds.

\section{Multiplexing GAin In MiMO DOWNLINK}

In this section we investigate the behavior of downlink throughput of a multiple-antenna cellular network at high transmit power regime and propose a lowerbound for the achievable multiplexing gain.

Consider a similar multi-cell network as in Fig. 1 with $K$ single-user cells. However, we assume each BS has $M$ antennas, each MS has $N$ antennas and every BS wishes to send $K$ different messages to $K$ MSs in $K$ cells. By having multiple antennas and allowing such a transmission scheme, the downlink throughput would be substantially increased.

We define the multiplexing gain of this network as [5]

$$
\eta \triangleq \lim _{\rho \rightarrow \infty} \frac{R_{N W}^{\operatorname{Sum}}(\rho)}{\log \rho}
$$

where $\rho$ is SNR and $R_{N W}^{\operatorname{Sum}}(\rho)$ is the network sumrate. As mentioned in Section I, the multiplexing gain represents the linear scaling of throughput over $\log S N R$ at high SNR.

In the information theory literature, this MIMO downlink with multiple messages for each user is called MIMO X channel [6] with $K$ transmitters and $K$ receivers. In [6], a lowerbound and an upperbound for the multiplexing gain of a 2-transmitter 2-receiver MIMO X channel has been established. It is shown that there are cases where the removal of some receive antennas does not affect the multiplexing gain. This raises a natural question that given $K$ and $M$, what is maximum multiplexing gain we can get and what is the corresponding optimal $N$. We will answer that question in the following.

Without loss of generality, we can assume the number of antennas at a BS is larger than the number of antennas at an MS. Suppose we are using the zero forcing transmission for every message. For the transmission of $K$ messages to $K$ users, the base station constructs one beamforming vector for each message such that it is orthogonal to the row space of the $(K-1) N$-by- $M$ interference matrix formed by the other $K-1$ transmissions. As a result, only $M-N(K-1)$ remaining antennas at each base station can be utilized for data streams. Effectively the zero forcing scheme creates $K$ MAC channels, each consists of $K$ transmitters and each transmitter has $M-N(K-1)$ antennas. The multiplexing gain of the MAC reads [5]:

$$
\eta_{M A C}(N)=\min \{K[M-N(K-1)], N\}
$$

Accordingly, the multiplexing gain of the $\mathrm{X}$ channel is:

$$
\begin{aligned}
\eta_{X}(N) & =K \times \eta_{M A C} \\
& =K \times \min \{K[M-N(K-1)], N\}
\end{aligned}
$$

Then we have the following theorem.

Theorem 1 The multiplexing gain of the MIMO X channel with $K M$-antenna transmitters and $K N$-antenna receivers is lower-bounded by:

$$
\eta_{X}^{*}=\max \left\{\eta_{X}\left(\left\lfloor N^{*}\right\rfloor\right), \eta_{X}\left(\left\lceil N^{*}\right\rceil\right)\right\}
$$

where $N^{*}=\frac{K M}{K^{2}-K+1}$. 
Proof: Consider (14), since $K[M-N(K-1)]$ is a decreasing function of $N$ with given $K$ and $M$, the optimal number of receive antennas at each receiver is

$$
N_{o p t}=\underset{N \in\left\{\left\lfloor N^{*}\right\rfloor,\left\lceil N^{*}\right\rceil\right\}}{\arg \max } \eta_{X}(N) .
$$

where

$$
\begin{aligned}
N^{*} & =\arg \{K(M-N(K-1))=N\} \\
& =\frac{K M}{K^{2}-K+1} .
\end{aligned}
$$

Accordingly, $\eta_{X}^{*}$ is achievable.

Applying this result to the 2-transmitter 2-receiver $\mathrm{X}$ channel $(K=2)$ we have $N^{*}=\frac{2 M}{3}$ and $\eta_{X}^{*}=$ $\max \left\{\eta_{X}\left(\left\lfloor\frac{2 M}{3}\right\rfloor\right), \eta_{X}\left(\left\lceil\frac{2 M}{3}\right\rceil\right)\right\}$. With $M=3$ we get the same multiplexing gain of 4 as the MMK scheme [7]. In general, when $M$ is a multiple of 3 , our achievable multiplexing gain coincides with the maximum multiplexing gain setup by Jafar in [6]. The above result is also applicable for networks with different number of transmitters and receivers.

\section{Conclusions}

In this paper we used an information theoretic approach to establish some lower and upper bounds on the downlink throughput of cellular networks. A lowerbound for the multiplexing gain in MIMO downlink with multiple messages at a base station is also established and shown to be optimal in 2transmitter 2-receiver case with certain number of antennas. The possible extensions of this work include investigating the interference channel with multiuser detection (IF-MUD). It is unknown that in a multiuser scenario, under what condition the MUD performs better than SUD and by how much. We may obtain a tighter lowerbound for the downlink throughput in the case MUD outperforms SUD. The problem can also be extended to the partial CSIT scenario.

\section{ACKNOWLEDGEMENT}

This work was supported by the Basic Research Program of the Korea Science and Engineering Foundation (R012006-000-11112-0) and was supported by the center for Broadband OFDM Mobile Access (BrOMA) at POSTECH through the ITRC program of the Korean MIC, supervised by IITA. (IITA-2006-C1090-0603-0037)

\section{REFERENCES}

[1] I. Sason, "On achievable rate regions for the Gaussian interference channels", IEEE Trans. Inform. Theory, vol. 50, pp. 1345-1356, June 2004.

[2] G. Kramer, "Outer bounds on the capacity of interference channels", IEEE Trans. Inform. Theory, vol. 50, pp. 581-586, March 2004.
[3] S. Vishwanath and S. Jafar, "On the Capacity of Vector Gaussian Interference Channels", ITW 2004, San Antonio, Texas, Oct. 2004.

[4] L. Zheng and D. N. Tse, "Diversity and Multiplexing: A Fundamental Tradeoff in Multiple-Antenna Channels", IEEE Trans. Inform. Theory, vol. 49, No.5 pp. 1073-1096, May 2003.

[5] S. Jafar, M. Fakhereddin, "Degrees of Freedom for the MIMO Interference Channel", Proc. IEEE Int. Symp. Information Theory (ISIT), pp. 1452-1456, July 2006.

[6] S. Jafar, "Degrees of Freedom on the MIMO X Channel Optimality of Zero Forcing and the MMK scheme", Preprint.

[7] M. Maddah-Ali, A. Motahari, and A. Khandani, "Signaling over MIMO multi-base systems: combination of multi-access and broadcast schemes", Proc. IEEE Int. Symp. Information Theory (ISIT), pp. 2104-2108, July 2006.

[8] T. M. Cover and J. A. Thomas, "Elements of Information Theory.", New York: Wiley, 1991.

[9] N. Jindal, "Multi-user Communication System: Capacity, Duality, and Cooperation", Ph.D. dissertation, Stanford University, July 2004.

[10] G. J. Foschini, K, Karakayali, and R. A. Valenzuela, "Coordinating Multiple Antenna Cellular Networks to Achieve Enormous Spectral Efficiency", IEE Proceedings - Communications, Volume 153, Issue 4, p. 548-555, August 2006.

[11] G. J. Foschini, G.J., Huang, H.C., Karakayali, K., Valenzuela, R.A., and Venkatesan, S., "The value of coherent base station coordination", Conf. on Information Science and Systems (CISS), Johns Hopkins University, Baltimore, MD, USA, March, 2005.

[12] M. Costa, "Writing on dirty paper", IEEE Trans. Inform. Theory, vol. 29, pp. 439-441, May 1983.

[13] S. Vishwanath, N. Jindal, and A. Goldsmith, "Duality, achievable rates, and sum-rate capacity of MIMO broadcast channels", IEEE Trans. Inform. Theory, vol. 49, no. 10, pp. 2658.2668, Oct. 2003.

[14] P. Viswanath and D. N. Tse, "Sum capacity of the vector Gaussian broadcast channel and uplink.downlink duality", IEEE Trans. Inform. Theory, vol. 49, no. 8, pp. 1912.1921, Aug. 2003.

[15] S. Vishwanath, N. Jindal, and A. Goldsmith, "On the capacity of multiple input multiple output broadcast channels", in Proc. Int. Conf. Communications, Apr. 2002, pp. 1444-1450.

[16] H. Weingarten, Y. Steinberg, and S. Shamai (Shitz), The capacity region of the Gaussian MIMO broadcast channel, Proc. IEEE Int. Symp. Information Theory (ISIT), pp. 174. June 2004.

[17] H. Viswanathan, S. Venkatesan, and H. Huang, "Downlink capacity evaluation of celluar networks with known-interference cancellation", IEEE J. Sel. Areas Commun., vol. 21, pp. 802811, June 2003.

[18] D. Wajcer, A. Wiesel and S. Shamai (Shitz), "Optimal beamforming for the Multi-antenna Gaussian broadcast channel using Superposition coding", in preperation.

[19] M. Kobayashi and G. Caire, "An Iterative Water-Filling Algorithm for Maximum Weighted Sum-Rate of Gaussian MIMOBC", IEEE J. Sel. Areas Commun., vol. 24, pp. 1640-1646, August 2006.

[20] H. Dai, A. F. Molisch, and H. Vincent Poor, "Downlink Capacity of Interference-Limited MIMO Systems With Joint Detection", IEEE Trans. Wireless Communications, vol. 3, pp. 442-453, March 2004. 